\title{
Uncovering the secrets to relieving stress: discrete element analysis of force chains in particulate media
}

\author{
Maya Muthuswamy* John Peters ${ }^{\dagger}$ \\ Antoinette Tordesillas ${ }^{\ddagger}$
}

(Received 8 November 2005; revised 24 August 2006)

\begin{abstract}
The traditional analyses for structures that are either built upon (for example, foundations), or are used for storage and handling of particulate solids (for example, bins, silos, hoppers, and bunkers) cannot account for important processes originating in the evolution of microscale particle kinematics and kinetics. Until recently, the complexity of these processes could only be perceived through macroscale
\end{abstract}

*Department of Mathematics and Statistics, University of Melbourne, 3010, Australia. mailto:mayadm@ms . unimelb.edu . au

${ }^{\dagger}$ U. S. Army Corps of Engineers, Engineer Research and Development Center, Vicksburg, U. S. A.

${ }^{\ddagger}$ Corresponding author: Department of Mathematics and Statistics, University of Melbourne 3010, Australia. mailto:atordesi@ms . unimelb.edu . au

See http://anziamj.austms.org.au/V47EMAC2005/Muthuswamy for this article, (C) Austral. Mathematical Soc. 2006. Published October 7, 2006. ISSN 1446-8735 
experiments, but information derived from these experiments lacks the necessary level of detail that is needed for sound theoretical development. Limited information on the evolution of microstructural properties in deforming particulate solids emerged through ingenious experiments using photoelastic techniques. These techniques revealed the complex microstructural fabric and force propagation, the evolution of which governs what is observed as the constitutive response of the material. More recently, numerical simulations using the discrete element method have also proven to be powerful tools - effectively providing a virtual laboratory for studying microstructural evolution. We use the discrete element technique to examine the propagation of forces within a particulate solid that is subject to an indenting rigid flat punch on its surface. Particular attention is given to the formation, evolution and collapse of "force chains" (that is, quasi-linear chains of load-bearing particles). These force chains are surrounded by a weak network of particles that, despite carrying only a small amount of the load, provides the necessary stability for such chains to persist. A mathematical framework for the characterisation of these chains is used to investigate the evolution of the force chain network with indentation, with a focus on microstructure, force chain lengths, and length distributions. Finally, the first steps toward establishing correlations across multiple length scales are made by linking the macroscopic load-penetration response to the underlying evolution of contact forces and force chains.

\section{Contents}

1 Introduction 


\section{Introduction}

Much of what is observed as the constitutive response (that is, stress-strain relation) of a particulate material is directly attributed to the underlying "microstructural evolution": changes in the particle kinematics and interparticle contact force transmission within the deforming medium. Microstructural evolution occurs across multiple length scales and has significant impact on macroscopic rheological behaviour. For example, experimental observations of key features on the mesoscale, such as finite width shear bands [9], along with efforts at their prediction through continuum based theory [16], clearly suggest micro, meso and macro scale dependencies in parameters. Thus, future models of particulate media must address physical mechanisms in both the microscopic (particle contact) level and the intermediate or mesoscopic domain (spanning several particles) and link these to the observable macroscopic behavior.

This article focuses on the nature of force propagation in a particulate material. Force propagation in particulate assemblies gives rise to chain-like regions of large forces known as "force chains" (see Figure 1). On the granular mesodomain, force propagation is characterised by the force chain network, which represents the main pathways of force transmission. Surrounding this highly ramified network is a weak network of particles that, despite carrying only a small amount of force, provides the necessary stability for such force chains to persist. As deformation proceeds, the force chain network undergoes rapid changes in branch morphology - reflecting the continual generation of new chains accompanied by the collapse of pre-existing chains.

Over the past decade, there has been intensive research devoted to the 

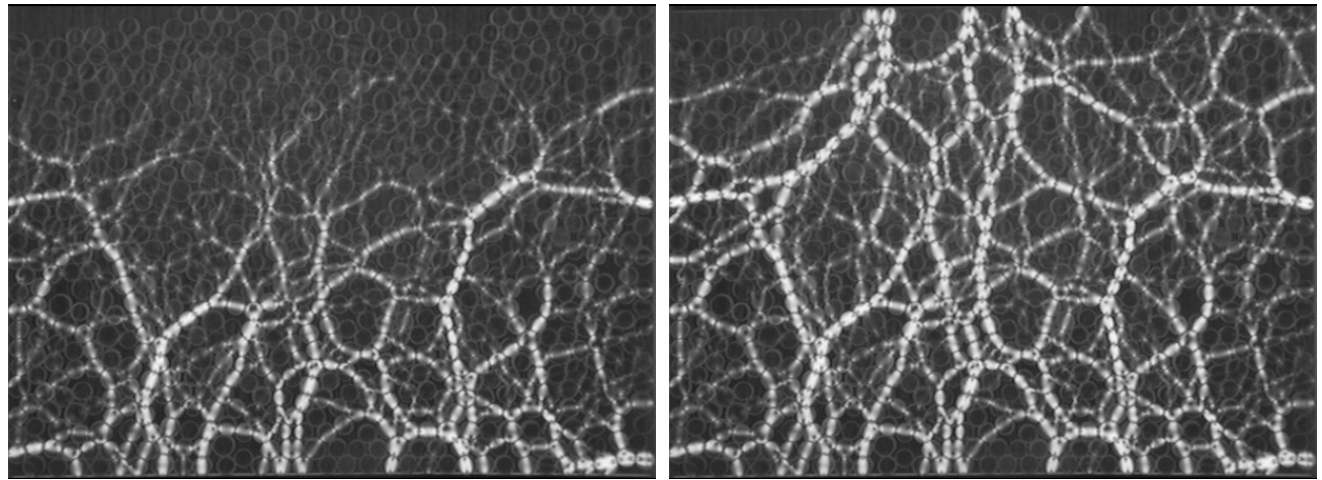

FigURE 1: Images of a photoelastic disk experiment of particles confined in a vertical column [3], showing effects of gravitational loading (left) prior to a uniform load applied to the top (right).

development of techniques for simulation and analysis of force chains as well as their validation against experimental results $[6,15,14,8$, e.g.]. The key ingredient in all of these studies is a quantitative measure of the contact force acting at each interparticle contact. From a theoretical viewpoint, the most effective tool for this purpose has been been the discrete element method which provides explicit information on the direction and magnitude of contact forces between particles. However, until recently, contact forces between particles could not be easily measured, and hence, experimental validation at this scale has not been performed. However, a recent breakthrough has been made whereby each individual vectorial contact force (both normal and tangential components) has been measured for a large two dimensional system of 2500 photoelastic disks [7]. This invaluable microstructural information thus provides a unique opportunity for validation of discrete element simulations and continuum models at the most fundamental level.

Once contact forces are established, a method must be developed to quantitatively identify or characterise force chains. In the past, the identification of force chains has been rather ad hoc and subjective [5]. Inspired by the 
innovation in [7], we recently proposed a mathematical framework for the characterisation of force chains [13]. Based on this, an algorithm was developed and implemented in discrete element simulations to establish the force chain network for a given particulate assembly at each stage of its deformation history. Here, a modified version of this algorithm examines the evolution of the resulting force chain network in a particulate material subject to an indenting rigid flat punch. The objective is to characterise the evolution of force chains as the punch indents. In particular, variations in the force chain properties including the average length of chains, total number of chains and distribution of chain lengths are established at various stages of the deformation history. Finally, first steps toward establishing correlations across multiple length scales are made by linking the macroscopic load penetration response to the underlying evolution of contact forces and force chains.

\section{Characterisation of force chains}

The proposed quantitative definition of a force chain is "a quasi-linear group of at least three particles with above average stress" [13]. This definition provides a quantitative method to characterise which particles are part of chains. Here, 'particle stress' refers to the largest eigenvalue, scaled by height to remove gravity effects, of a tensorial force moment per particle [1], which gives an indication of the load carried by the particle.

Quasi-linear is quantified by minimising the angle between branch vectors joining a pair of particles and the eigenvector corresponding to the particle stress. Once particles are allocated to chains, various properties of a chain can be calculated. For example, one property used in the following results is the average 'strength' of a chain, which is the average of all the particle 'stresses' in the chain. Peters et al. [13] give a more complete discussion of this analysis. 
TABLE 1: discrete element method parameters and material properties.

\begin{tabular}{ll}
\hline Total indentation (percent) & 14 \\
Time step increment & $2.86 \times 10^{-6} \mathrm{~s}$ \\
Punch velocity & $1.02 \times 10^{-2} \mathrm{~m} / \mathrm{s}$ \\
Particle density & $2650 \mathrm{~kg} / \mathrm{m}^{3}$ \\
Radius distribution min & $0.846 \times 10^{-3} \mathrm{~m}$ \\
Radius distribution max & $2.54 \times 10^{-3} \mathrm{~m}$ \\
Inter-particle friction coefficient & 0.5 \\
Particle-punch friction coefficient & 0.0 \\
Rolling friction coefficient & 0.2 \\
Normal stiffness constant & $1.0 \times 10^{5} \mathrm{~N} / \mathrm{m}$ \\
Tangential stiffness constant & $0.2 \times 10^{5} \mathrm{~N} / \mathrm{m}$ \\
Rotational stiffness constant & $2.26 \times 10^{-1} \mathrm{Nm} / \mathrm{rad}$ \\
\hline
\end{tabular}

\section{$3 \quad$ Numerical experiment}

The system considered is a 2D polydisperse assembly of 10850 circular disks subject to indentation by a rigid flat punch. The discrete element model used is derived from earlier work by Horner et al. [4]. It has been partially validated against photoelastic disk experiments, via distributions of contact force magnitudes and directions $[12,2,10]$. Simple contact laws based on Hooke's law in conjunction with Coulomb's law are used. The initial configuration was created by dropping the particles into the box, and allowing them to settle until individual rotations and displacements became negligible (see Figure 2). After settling, the punch moved down to reach a total indentation of approximately $14 \%$ of the height of the box. Table 1 summarises key parameters used in the simulations. 


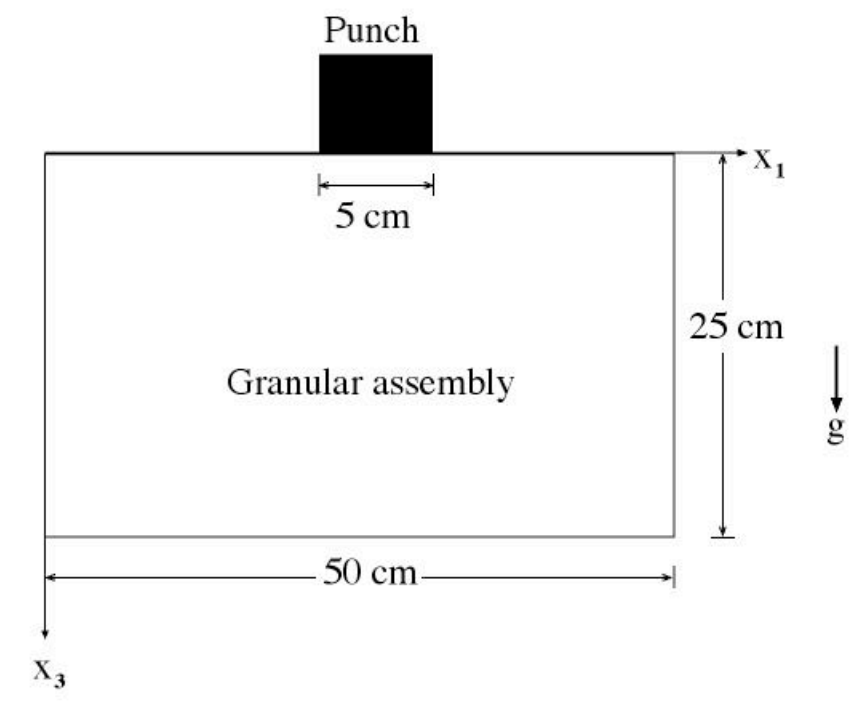

Average kinetic energy

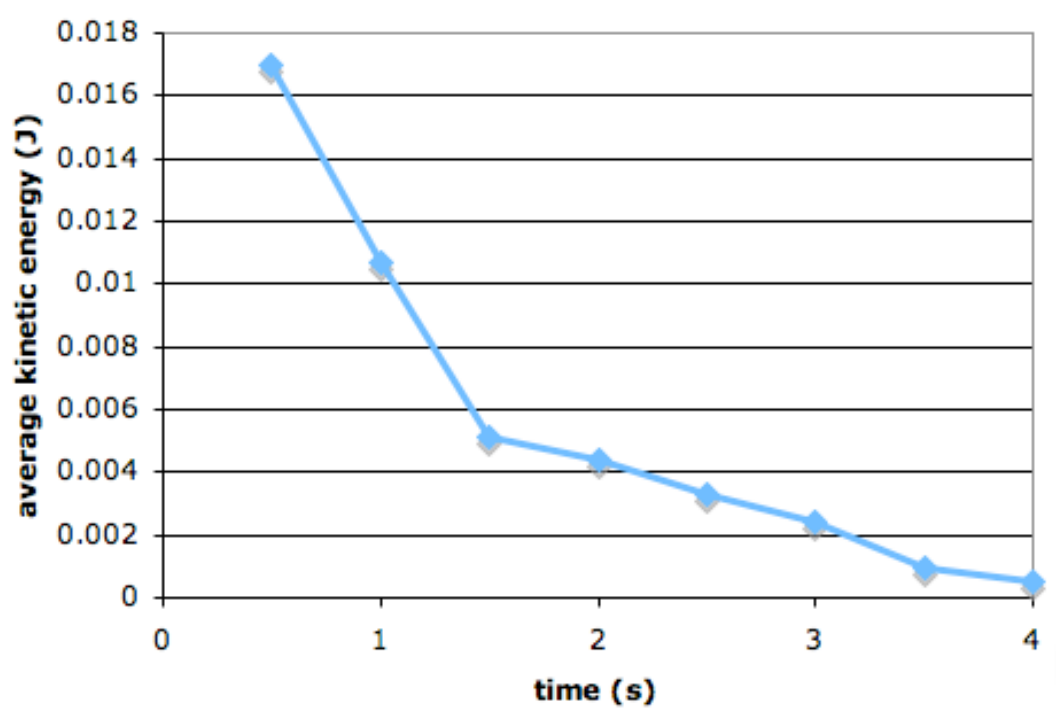

Figure 2: The initial setup and dimensions of the flat punch system (top), and average kinetic energy of particles during settling phase (bottom). 


\section{Results and discussion}

Figure 3 shows the system before the punch has indented. On the top, particles are coloured by the magnitude of their particle stress (defined in Section 2). The inhomogeneity of the stress field is clear, as are the preferential pathways of force transmission. On the bottom, particles are coloured according to whether or not they are part of a force chain, with the colour of each chain/particle representing its average strength (defined in Section 2). In all of the following results, dark blue corresponds to 0 (that is, not in a chain), then from light blue to red represents small to large strengths. A comparison of these two figures shows good agreement, demonstrating that the particle stress allows a characterisation of both direction and magnitude of force chains.

Figure 4 shows the evolution of the force chain network under the punch, for three stages in indentation (referred to as stages A, B and C). As the punch moves into the material, force chains form to support the applied load, emanating radially outwards from the base of the punch.

An important application of this method of characterising force chains is the ability to measure individual force chain lengths, which has previously only been done in an ad hoc fashion in experiments [5]. Figure 5 (top) shows the evolution of the average length of force chains in two different averaging regions (over the whole specimen and in a smaller region under the punch). When averaged over the region under the punch, chains are slightly longer than when averaged over the entire specimen, which can be qualitatively observed in Figure 4. Interestingly, as the punch indents, there is no noticeable change in the average chain length for either averaging regions, which suggests a characteristic force chain length. Figure 5 (bottom) shows how the distribution of chain lengths changes with evolution for stages $\mathrm{A}-\mathrm{C}$. The inset shows a semi-log plot, demonstrating an exponential distribution for lengths up to 11 particles, for all stages, consistent with experimental findings [5]. Once again, as there is no discernible change in the distribution 

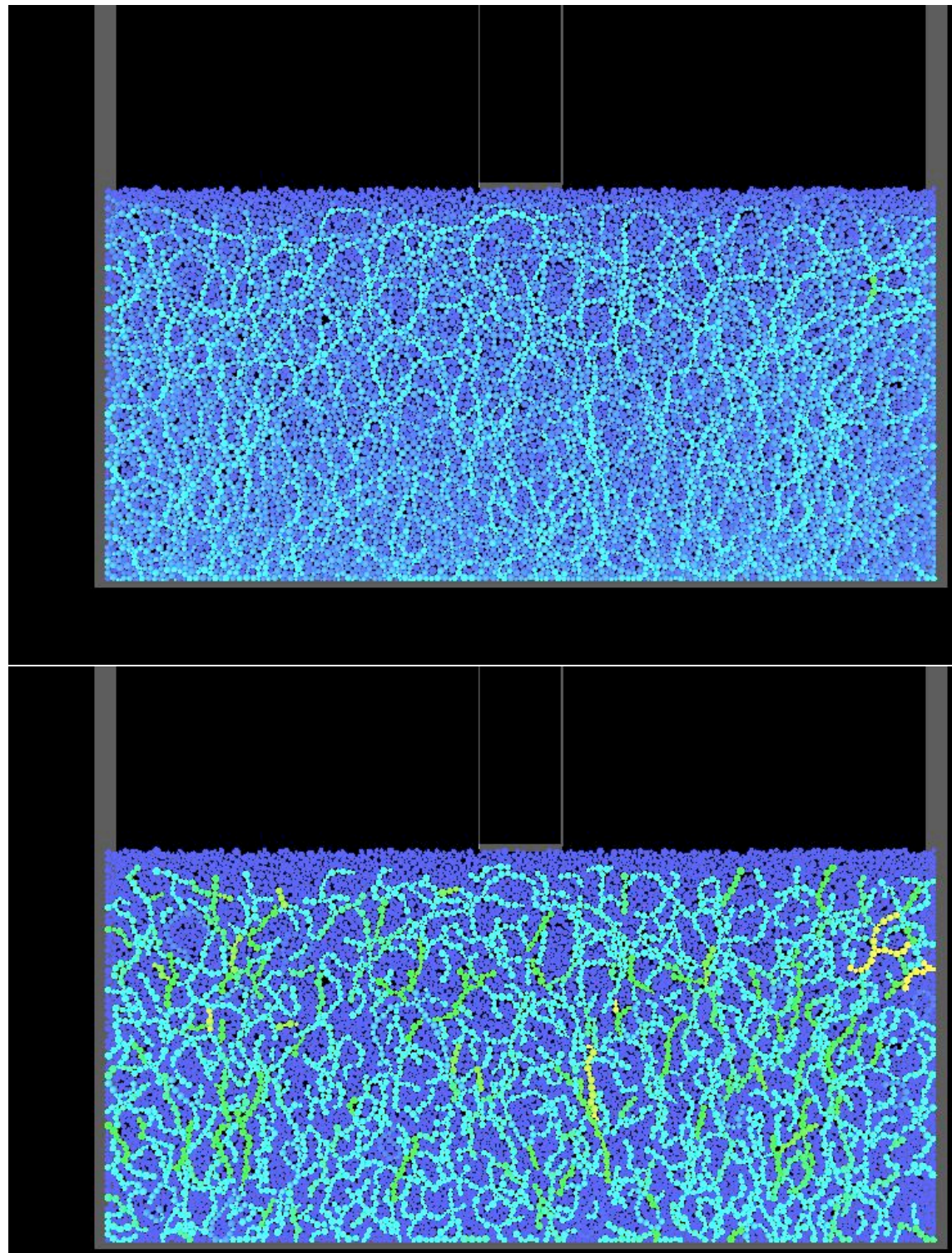

FigurE 3: Comparison of particle stresses (top) with force chains (bottom), before the punch has indented. 


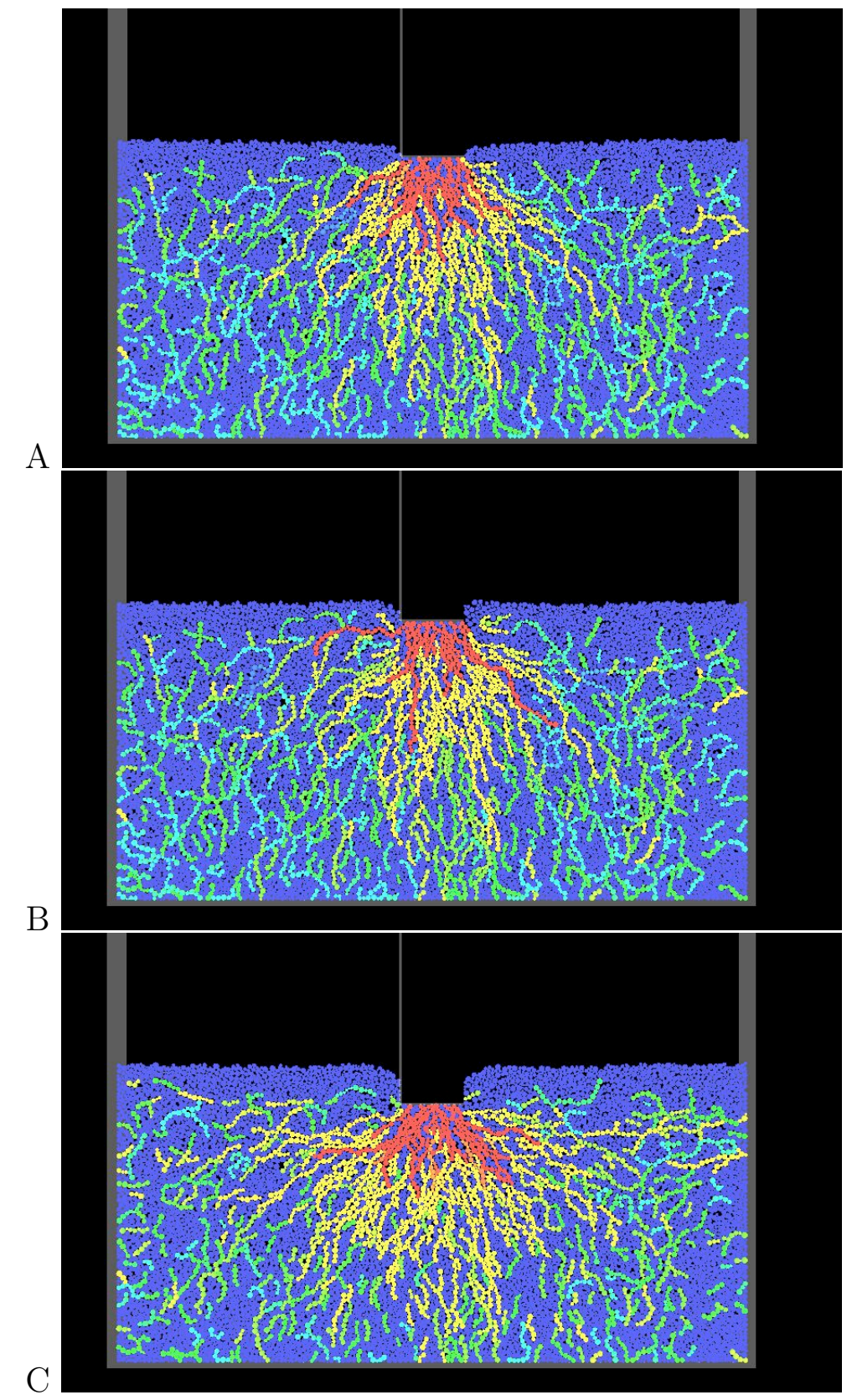

Figure 4: The force chain network at three stages of indentation: stages A (top), B (middle) and C (bottom). 
with indentation, this is further evidence of a characteristic length.

Figure 6 (top) shows the percentage of all particles that are part of force chains, for the same two averaging regions used in Figure 5. Initially, this percentage is the same for the two averaging regions: since the punch has not indented yet, the spatial distribution of chains is isotropic (see Figure 3). However, as the punch indents, the percentage in force chains increases in the region under the punch, but decreases when averaged over the whole specimen. Again, this may be qualitatively observed in Figure 4. By varying the size of the averaging region and measuring when this quantity converges, the extent of the plastic flow zone could be established.

Figure 6 (bottom) shows the macroscopic quantity of load penetration curve for the material. Stages A-C are marked on the graph. The sudden drop in load observed macroscopically is explained via a closer inspection of Figure 4. At stage B (after the drop), the force chain network appears to have diffused and become weaker. To examine this drop, let us focus on what happens to only those particles that are part of major chains at stage A (we choose the $12 \%$ of chains at stage A with highest strength). Prior to stage A (at $5.3 \%$ indentation), $89 \%$ of the major chains were part of a chain, with $82 \%$ of these experiencing a build up in stress in the lead up to stage A. This demonstrates that most of the major chains were initially present in the existing force chain structure and strengthen on applied load. As indentation proceeds from $\mathrm{A}$ to $\mathrm{B}$, the force chain network reconfigures: orientations of many of the major chains at A are no longer optimal pathways of force transmission. These break down and new chains are formed to resist the punch more efficiently. This reconfiguration of the force chain network is evident in Figures 4, 6 and 7. In particular, Figure 7 indicates that of the major chains at $\mathrm{A}, 70 \%$ have experienced a sudden reduction in strength from $\mathrm{A}$ to $\mathrm{B}$. Further, of these, $30 \%$ have actually collapsed by stage B (corresponding to particles no longer in force chains). Stage B to C corresponds to the new force chain network created at B undergoing the same process as the original structure from the start to stage A. Note that in Figure 7 and in the 


\section{Average force chain length}

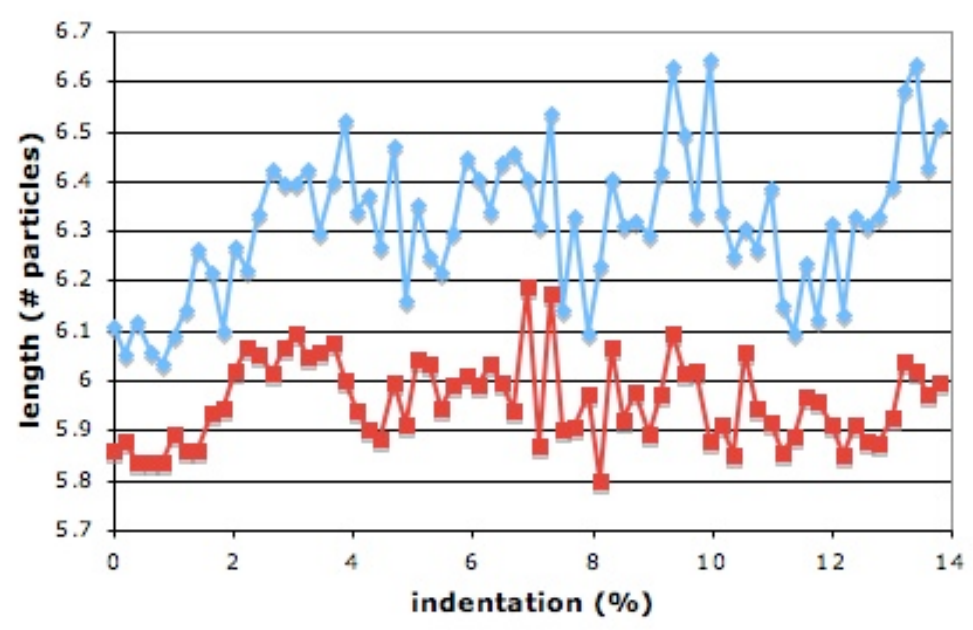

$\multimap$ Under punch - Whole specimen

\section{Distribution of chain lengths}

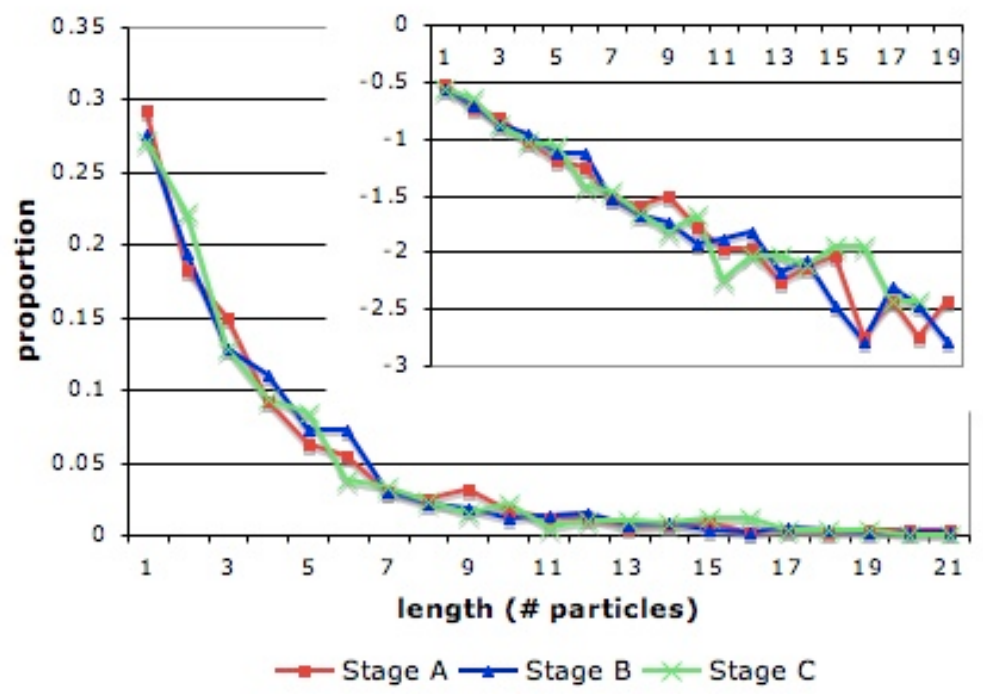

Figure 5: Evolution of the average length of force chains, for the two different averaging regions (top), and length distributions for stages $\mathrm{A}-\mathrm{C}$, with inset showing a log plot (bottom). 


\section{Percentage of particles in force chains}

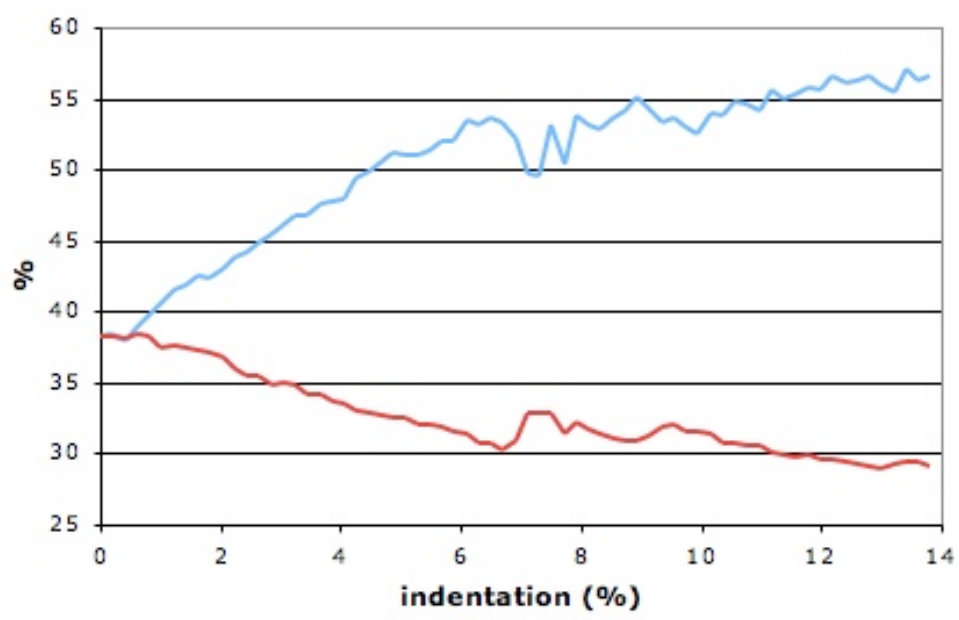

Under punch —Whole specimen

Load-penetration curve

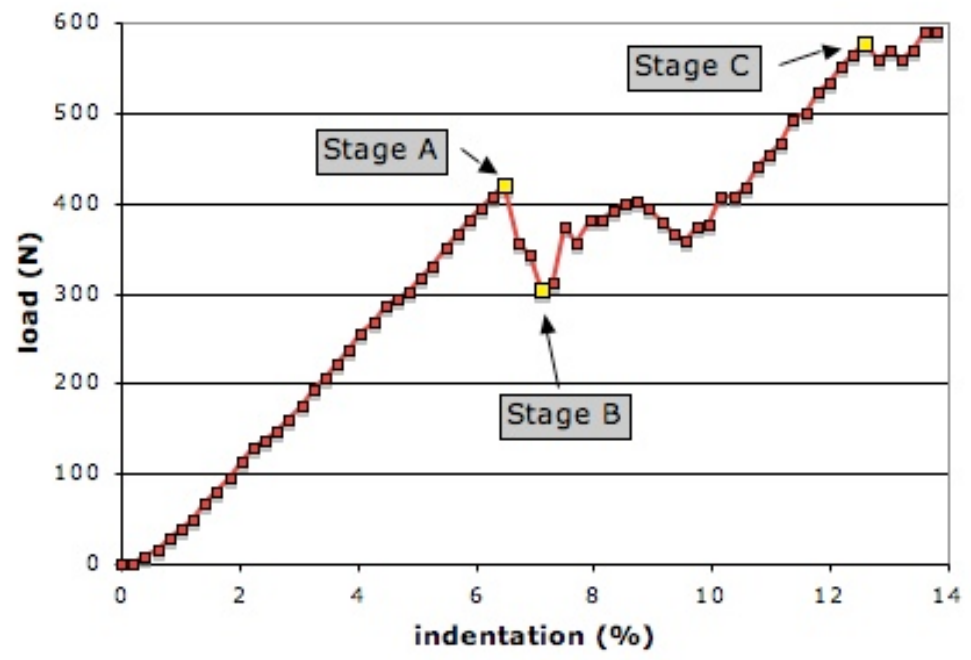

FIGURE 6: Evolution of the percentage of particles that are in force chains, for the two different averaging regions (top) and bulk property of loadpenetration curve, with the three stages in indentation marked (bottom). 


\section{Correlation between strengths at stages $A$} and $B$

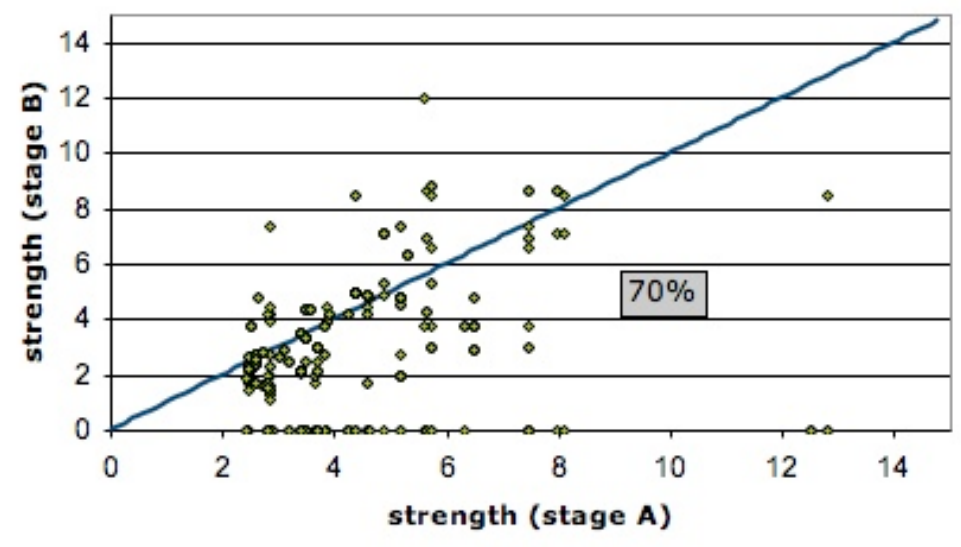

Figure 7: Correlation between strengths of major force chains at stage A and stage B.

preceding discussion, we only focused on what happens to a reference group of particles (major chains at stage A). Finally, the drop in load appears correlated with fluctuations observed in the percentage of particles in chains (Figure 6). This provides motivation for a more comprehensive investigation of force propagation across three length scales (that is, contact force at microscale, force chain at mesoscale, and load penetration at macroscale) to identify and quantify correlations between behaviour across these scales [11].

\section{Conclusions}

The key findings of this research are: 
1. Using the method for characterising force chains [13], the evolution of the force chain network of a granular material under indentation by a rigid flat punch was investigated.

2. Force chains were found to be longer directly underneath the punch (approximately 6.3 particles) than further away from the punch (approximately 5.9 particles), and more particles are in force chains directly underneath the punch than further away.

3. The distribution of chain lengths was found to be exponential, consistent with experiments [5].

4. Neither the average length nor the distribution of force chains varied noticeably with indentation, suggesting a characteristic force chain length scale.

5. The macroscopic quantity of load penetration curve was examined, and the connection between its fluctuations and the force chain network discussed. The sudden drop in load was due to the reduction in strength of pre-existing chains, with some to the point of collapse. This correlation is evidence of bulk behaviour being closely governed and controlled by events occurring at smaller scales (inter-particle contact force on the microscale and force chain on the mesoscale).

This investigation paves the way for a more comprehensive multiscale analysis to quantify and explain these correlations across three length scales [11].

Acknowledgements Professor Robert Behringer is gratefully acknowledged for supplying Figure 1 [3]. The support of the US Army Research Office through a grant to AT (grant number DAAD19-02-1-0216) and the Australian Research Council (DP0558808) through a grant to AT is gratefully acknowledged, as is the University of Melbourne Advanced Research 
Computing section through a grant of computer time. This paper also reports on research conducted under the AT22 Research Project Stress Transfer in Granular Media conducted at the U.S. Army Engineer Research and Development Center (ERDC). Permission was granted by the Director of the Geotechnical and Structures Laboratory, ERDC to publish this information. MM gratefully acknowledges the support of the Pratt Foundation Scholarship for postgraduate study.

\section{References}

[1] J. T. Bosko and A. Tordesillas. Unjamming transitions in dense granular assemblies subjected to biaxial compression. Phys. Rev. E, submitted. C359

[2] H. S. Hafez. 600-311 research report: A discrete element simulation of a 2D assembly of bi-disperse circular disks subjected to pure shear. Technical report, The University of Melbourne, 2005. C360

[3] R. R. Hartley. Evolving force networks in deforming granular materials. PhD thesis, Duke University, 2003. C358, C369

[4] D. A. Horner, J. F. Peters, and A. Carrillo. Large scale discrete element modeling of vehicle-soil interaction. J. Eng. Mech.-ASCE, 127(10):1027-1032, 2001. doi:10.1061/(ASCE)0733-9399(2001)127:10(1027) C360

[5] D. Howell, R. P. Behringer, and C. Veje. Stress fluctuations in a 2D granular couette experiment: A continuous transition. Phys. Rev. Lett., 82(26):5241-5244, 1999. doi:10.1103/PhysRevLett.82.5241 C358, C362, C369 
[6] S. Luding. Stress distribution in static two-dimensional granular model media in the absence of friction. Phys. Rev. E, 55(4):4720-4729, 1997. doi:10.1103/PhysRevE.55.4720 C358

[7] T. S. Majmudar and R. P. Behringer. Contact force measurements and stress-induced anisotropy in granular materials. Nature, 435(7045):1079-1082, 2005. doi:10.1038/nature03805 C358, C359

[8] H. A. Makse, D. L. Johnson, and L. M. Schwartz. Packing of compressible granular materials. Phys. Rev. Lett., 84(18):4160-4163, 2000. doi:10.1103/PhysRevLett.84.4160 C358

[9] H.-B Muhlhaus and I. Vardoulakis. The thickness of shear bands in granular material. Geotechnique, 37(3):271-283, 1987. C357

[10] M. Muthuswamy and A. Tordesillas. Micro-level validation of discrete element simulations with experiments: a focus on force propagation, 2006. Poster presentation at Fifth World Congress on Particle Technology. C360

[11] M. Muthuswamy and A. Tordesillas. How do interparticle contact friction, packing density and degree of polydispersity affect force propagation in particulate assemblies? J. Stat. Mech. Theory Exp., P09003, 2006. doi:10.1088/1742-5468/2006/09/P09003 C368, C369

[12] N. Olshina. 620-312 research project: Contact forces in 2D bidisperse assembly of circular disks subject to isotropic compression: A DEM investigation. Technical report, The University of Melbourne, 2005. C360

[13] J. F. Peters, M. Muthuswamy, J. Wibowo, and A. Tordesillas. Characterization of force chains in granular material. Phys. Rev. E, 72(4):041307, 2005. doi:10.1103/PhysRevE.72.041307 C359, C369 
[14] F. Radjai, D. E. Wolf, M. Jean, and J. J. Moreau. Bimodal character of stress transmission in granular packings. Phys. Rev. Lett., 80(1):61-64, 1998. doi:10.1103/PhysRevLett.80.61 C358

[15] S. Schollmann. Simulation of a two-dimensional shear cell. Phys. Rev. E, 59(1):889-899, 1999. doi:10.1103/PhysRevE.59.889 C358

[16] A. Tordesillas, J. F. Peters, and B. S. Gardiner. Insights into 1D localisation theory and micromechanical constitutive laws. Geotechnique, 54(5):327-330, 2004. doi:10.1680/geot.54.5.327.46727 C357 